\title{
Le maraîchage périurbain à Libreville et Owendo (Gabon): pratiques culturales et durabilité
}

\author{
Sandrine Mariella Bayendi Loudit ${ }^{1,2, *}$, Auguste Ndoutoume Ndong ${ }^{2}$ et Frédéric Francis ${ }^{1}$ \\ ${ }^{1}$ Entomologie fonctionnelle et évolutive, Gembloux Agro-Bio Tech, université de Liège, 2 passage des déportés, 5030 Gembloux, \\ Belgique \\ ${ }^{2}$ Institut de recherches agronomiques et forestières, BP 2246, Libreville, Gabon
}

\begin{abstract}
Résumé - Au Gabon, le rôle du maraîchage périurbain est essentiel à l'approvisionnement des villes en légumes frais, notamment à Libreville et Owendo. Lors d'une enquête menée dans trois périmètres maraîchers, un état des lieux a été réalisé sur les caractéristiques socio-économiques, la diversité des plantes cultivées et les pesticides utilisés. Les superficies exploitées vont de 0,08 ha à 0,4 ha par maraîcher selon les sites. Les exploitants interrogés sont à $51 \%$ originaires du Gabon alors que $40 \%$ sont originaires du Burkina Faso. Les espèces les plus cultivées tout au long de l'année sont l'amarante (Amaranthus hybridus L.), la laitue (Lactuca sativa L.), l'oseille de guinée (Hibiscus sabdariffa L.), la morelle noire (Solanum nigrum L.). Les ravageurs les plus importants sont les Aphididae et quelques coléoptères. Les produits phytopharmaceutiques les plus utilisés sont des insecticides neurotoxiques conventionnels. Une meilleure maîtrise du suivi des cultures, une vulgarisation en protection des cultures y compris sur l'emploi des pesticides ainsi que la possibilité de développer des systèmes de micro-crédits aux petits producteurs permettraient d'augmenter la production légumière périurbaine et sa qualité sanitaire, ainsi que d'accroître l'autonomie alimentaire locale.
\end{abstract}

Mot clés : légumes / maraîchage / Afrique centrale / ravageur / pesticide

\begin{abstract}
Peri-urban market gardening in Libreville and Owendo (Gabon): farmers' practices and sustainability. In Gabon, peri-urban gardening is an opportunity to provide vegetables to the main cities, such as Libreville and Owendo. Following a survey conducted in three market gardening areas, an inventory was conducted on the socio-economic characteristics, the diversity of crops, and pesticide uses. The cropped areas range from 0.08 ha to 0.4 ha per farmer, according to the site. National operators represent 51\%, while people from Burkina Faso manage $40 \%$ of vegetable production. The most cultivated species throughout the year are amaranth (Amaranthus hybridus L.), lettuce (Lactuca sativa L.), Guinea sorrel (Hibiscus sabdariffa L.) and black nightshade (Solanum nigrum L.). The most important pests are Aphididae and some beetles. The most commonly used plant protection products are insecticides, mainly conventional neurotoxic. Best crop monitoring, pest control including pesticide application reduction, and the possibility to offer microcredit systems to small producers would help increasing peri-urban healthy vegetable production and increase local food autonomy.
\end{abstract}

Keywords: vegetables / market gardening / Central Africa / pest / pesticide

\section{Introduction}

L'agriculture périurbaine est un atout pour assurer la sécurité alimentaire des pays en voie de développement, en particulier ceux de l'Afrique subsaharienne. En effet, les populations urbaines africaines croissent rapidement. Pour Temple et Moustier (2004), cette croissance pourrait encore

\footnotetext{
*Auteur de correspondance : sbayendiloudit@gmail.com
}

s'accélérer dans les 20 prochaines années et induire un besoin d'approvisionnement alimentaire régulier, basé sur les denrées importées et/ou produites par l'agriculture locale. Parmi ces produits alimentaires, les légumes occupent une place importante pour les populations urbaines dont ils satisfont non seulement les besoins en vitamines, en protéines et en autres éléments essentiels à la santé, mais à qui ils procurent également une diversité d'activités agricoles et des revenus substantiels (James et al., 2010). 


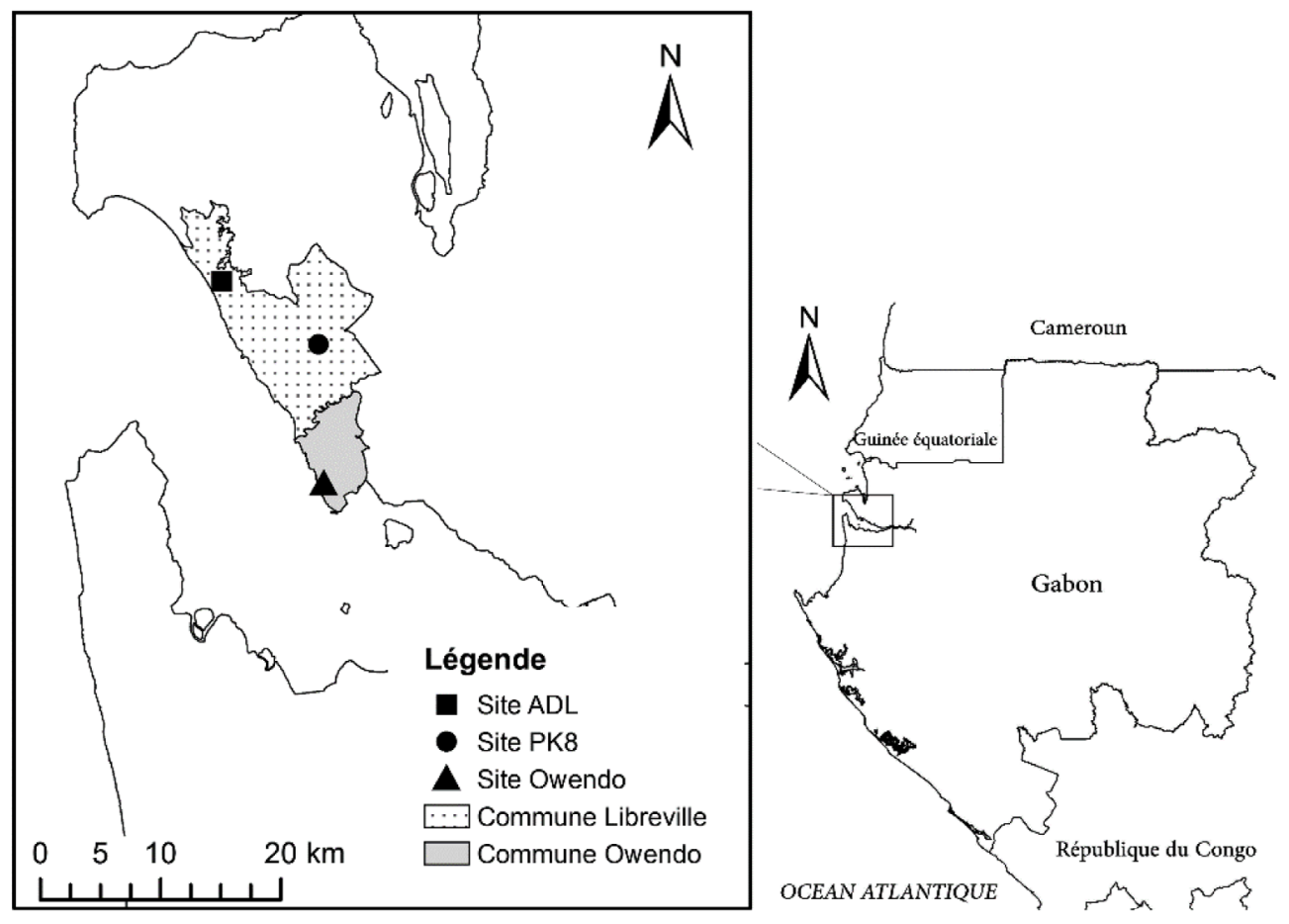

Source: Adaptée de Nguema (2005) et de WRI (World Resources Institute), 2013.

Source: Adapted from Nguema (2005) and WRI (World Resources Institute), 2013.

Fig. 1. Localisation des périmètres maraîchers.

Fig. 1. Market garden localization.

Cette réalité est aussi celle du Gabon, pays de 1,7 millions d'habitants, dont $87 \%$ d'urbains (Lefort, 2015). Si, sur une superficie totale de $267667 \mathrm{~km}^{2}$, près de 15,2 millions d'hectares sont considérés comme cultivables, seulement 495000 hectares sont effectivement exploités. Avec un secteur agricole peu développé, la satisfaction des besoins alimentaires est donc essentiellement tributaire des importations d'aliments (Organisation mondiale du commerce, 2013a).

En 2013, $60 \%$ des aliments consommés, essentiellement destinés aux populations urbaines, ont été importés (Organisation mondiale du commerce, 2013b). La FAO (Organisation des Nations Unies pour l'alimentation et l'agriculture) rapporte que le Gabon a un rendement de 5,5 t/ha en légumes frais, le plus bas de la zone CEMAC (Communauté économique et monétaire de l'Afrique centrale). En 2013, les importations se sont chiffrées à 572 tonnes, soit 18 fois la quantité de légumes frais importée par le Congo voisin, près de trois fois plus peuplé. Le Gabon représente ainsi $33 \%$ des importations légumières d'Afrique centrale (FAO, 2015). En raison d'une agriculture essentiellement tournée vers l'autoconsommation et du mauvais état du réseau routier intérieur, la production agricole des zones rurales ne contribue que faiblement à l'approvisionnement des marchés urbains. Les autres contraintes du développement de ce secteur sont la décroissance et le vieillissement de la population rurale, ainsi que l'absence de services techniques d'appui et de mécanismes d'accès au crédit. La seule politique agricole visible se limite à la timide relance des filières café et cacao. Il existe pourtant un tissu d'activités maraîchères urbaines et périurbaines qui pourrait être développé. C'est un des mandats de l'Institut gabonais d'appui au développement (IGAD, 2014). Afin de lui apporter une bonne connaissance des pratiques de maraîchers et d'étudier les solutions possibles aux problèmes qu'ils rencontrent, un inventaire des productions maraîchères et de la gestion des bio-agresseurs a été menée.

\section{Méthodologie}

\subsection{Localités d'étude}

Ce travail a été effectué au sein des trois périmètres maraîchers de l'IGAD, dans la province de l'Estuaire (Gabon), dans les communes de Libreville et d'Owendo (Fig. 1). Les périmètres de Libreville sont respectivement celui de l'ADL (l'aéroport de Libreville) $\left(0^{\circ} 27^{\prime} 30^{\prime \prime} \mathrm{N}, 9^{\circ} 25^{\prime} 06^{\prime \prime} \mathrm{E}\right)$ qui comporte 64 parcelles de $1000 \mathrm{~m}^{2}$ de superficie unitaire, et celui du PK8 $\left(0^{\circ} 24^{\prime} 39^{\prime \prime} \mathrm{N}, 9^{\circ} 29^{\prime} 26^{\prime \prime} \mathrm{E}\right)$ qui est constitué de 16 parcelles de $800 \mathrm{~m}^{2}$. Celui de la commune d'Owendo est situé au sud de Libreville $\left(0^{\circ} 18^{\prime} 26^{\prime \prime} \mathrm{N}, 9^{\circ} 29^{\prime} 40^{\prime \prime} \mathrm{E}\right)$ et comporte 27 parcelles de $1000 \mathrm{~m}^{2}$.

Le climat local est de type équatorial chaud et humide, avec des températures variant entre $22^{\circ} \mathrm{C}$ et $32^{\circ} \mathrm{C}$ et une pluviométrie annuelle de 1500 à $3000 \mathrm{~mm}$. On distingue quatre saisons :

- une petite saison sèche de janvier à février;

- une grande saison des pluies de mars à juin; 
- une grande saison sèche de juillet à septembre;

- une petite saison des pluies d'octobre à décembre (Direction générale des statistiques, 2010).

Le choix des sites a été orienté par la facilité d'accès aux parcelles, la stabilité des exploitants, ainsi que la formation et l'encadrement effectués par l'IGAD, structure paraétatique de formation et d'appui aux activités agricoles urbaines et périurbaines (IGAD, 2014).

\subsection{Enquêtes sur les exploitations maraîchères}

Cette recherche a été conduite durant la période allant de septembre à octobre 2011 chez 35 exploitants, ce nombre correspondant à $53 \%$ des maraîchers installés sur les sites de l'IGAD dans les communes de Libreville et Owendo. La part des exploitants interrogés par site est de $48 \%$ à ADL, $67 \%$ à Owendo et $50 \%$ au PK8. Des enquêtes ont été conduites chez les exploitants ayant consenti à être interrogés et favorables à la visite de leurs parcelles. Les données ont été collectées suivant un questionnaire portant sur des informations relatives aux caractéristiques socio-économiques des producteurs, à la diversité des systèmes de production et des plantes cultivées, ainsi qu'à la problématique des ravageurs rencontrés et des produits phytosanitaires utilisés. Ces données ont été dépouillées manuellement puis analysées avec le logiciel Excel. Toutes les proportions sont calculées par rapport au nombre total des interviewés. Une comparaison entre les différents sites a été effectuée.

\section{Résultats et discussions}

\subsection{Caractéristiques socio-économiques des maraîchers}

\subsubsection{Caractéristiques des maraîchers}

Les tranches d'âge des personnes interrogées varient entre 20 et 60 ans (Tab. 1 et Annexe 1). La population des jeunes maraîchers (20-35 ans) est inférieure (37\%) à celle de plus de 36 ans (63\%). Le vieillissement des exploitants pourrait poser un problème dans quelques années si la relève n'est pas assurée. Il faudrait donc inciter les jeunes à s'intéresser à l'agriculture. La proportion des hommes (83\%) est plus importante que celle des femmes (17\%). Cependant, sur le site d'Owendo la proportion des femmes de nationalité gabonaise atteint $36 \%$, ce qui montre l'intérêt possible des femmes pour cette activité. Cette proportion est supérieure à celle rencontrée, par exemple, dans les périmètres maraîchers au nord du Cameroun : $15 \%$ à Ngaoundéré et $11 \%$ à Bocklé (Nchoutnji et al., 2009). Les exploitants nationaux représentent $46 \%$ du total à Libreville et $64 \%$ à Owendo; ces proportions sont plus élevées que celles rapportées par Ondo (2011) qui indiquait $22 \%$ à Libreville. Cette différence peut être due au fait que notre échantillonnage s'est limité aux sites maraîchers de l'IGAD, puisque cet institut privilégie les nationaux pour l'affectation des parcelles. Concernant les étrangers, les originaires du Burkina Faso (respectivement $42 \%$ à Libreville et $100 \%$ à Owendo) sont les plus nombreux.

Il faut noter que, sur le site du PK8, seuls quelques nationaux ont accepté de participer à l'enquête; les autres craignaient qu'il ne s'agisse d'un contrôle.
Pour ce qui est du niveau d'instruction des maraîchers, plus de la moitié sont instruits. Cependant, il y a $34 \%$ d'illettrés ; cela rend plus difficile la conduite des cultures car toutes les informations concernant le suivi et l'entretien des plantes sont écrites.

La durée de pratique de l'agriculture est variable et va de zéro à 20 ans. La plupart des interviewés sont issus de familles d'agriculteurs. Soixante-six pour cent ont suivi une formation dispensée par les techniciens de l'IGAD. Pour les expatriés $(34 \%)$ qui ont des barrières de langue, mais qui possèdent néanmoins des notions en suivi des cultures, ce sont leurs camarades, installés depuis un certain temps sur les sites, qui se chargent de leur expliquer les recommandations de l'institut. De façon générale, l'IGAD se réserve le droit de sanctionner tout maraîcher qui n'assure pas un bon suivi de ses cultures.

\subsubsection{Habitat et personnes à charge}

On note que 63 \% des maraîchers sont locataires. Les types d'habitations sont variables: en dur (parpaings), à moitié en dur (soubassement en parpaing et planches par-dessus) et en planches. Certains exploitants $(31 \%)$ n'ont pas de personnes à charges. Ce sont pour la plupart des expatriés, qui viennent d'abord seuls. C'est lorsque leurs conditions de vie s'améliorent qu'ils sont rejoints par leurs familles. Pour les autres, la moyenne des enfants à domicile est de deux. Il faut noter que, de façon générale, plusieurs neveux, frères et sœurs sont à la charge des exploitants, même s'ils n'habitent pas dans le même domicile. Soixante-six pour cent des exploitants sont en couple; les conjoints sont un atout pour le travail au champ et/ou pour le commerce.

\subsubsection{Structure de commercialisation et revenus}

La commercialisation des légumes des maraîchers se fait selon trois façons. La vente sur le site est la plus importante : $94 \%$ des maraîchers. Six pour cent écoulent leurs produits chez des particuliers. Parmi ceux qui vendent sur le site, $76 \%$ ont des contrats tacites avec des commerçants grossistes qui, pour la plupart, viennent récupérer les produits au champ.

Rares sont ceux qui commercialisent leurs produits au marché ; cette tâche est réservée à une épouse ou à un enfant. On note que la moitié des exploitants $(51 \%)$ ont un revenu mensuel supérieur au salaire minimum interprofessionnel garanti (150000 FCFA, soit $229 €)$. Ce revenu est essentiellement lié aux activités agricoles. Vingt-trois pour cent des interviewés ont refusé de donner des informations sur leurs revenus. Le maraîchage a permis à $37 \%$ des exploitants d'être propriétaires de leurs habitations. D'autres activités annexes (gardien de nuit, taxi...) sont pratiquées par $34 \%$ des interviewés. Ils ont cependant refusé de donner des chiffres sur ces revenus.

\subsection{Caractéristiques des exploitations maraîchères}

\subsubsection{Accès au foncier et superficie exploitée}

L'IGAD met à la disposition des nationaux motivés, des parcelles gratuites pour leur permettre de développer l'activité maraîchère. La répartition avait été faite à la mise en place des périmètres maraîchers. Trois associations de maraîchers nationaux ont été créées pour permettre une meilleure gestion des sites. Lorsque certaines parcelles ne sont pas exploitées ou 
Tableau 1. Caractéristiques socio-économiques des maraîchers de Libreville et d'Owendo.

Table 1. Socio-economic characteristics of market gardeners of Libreville and Owendo.

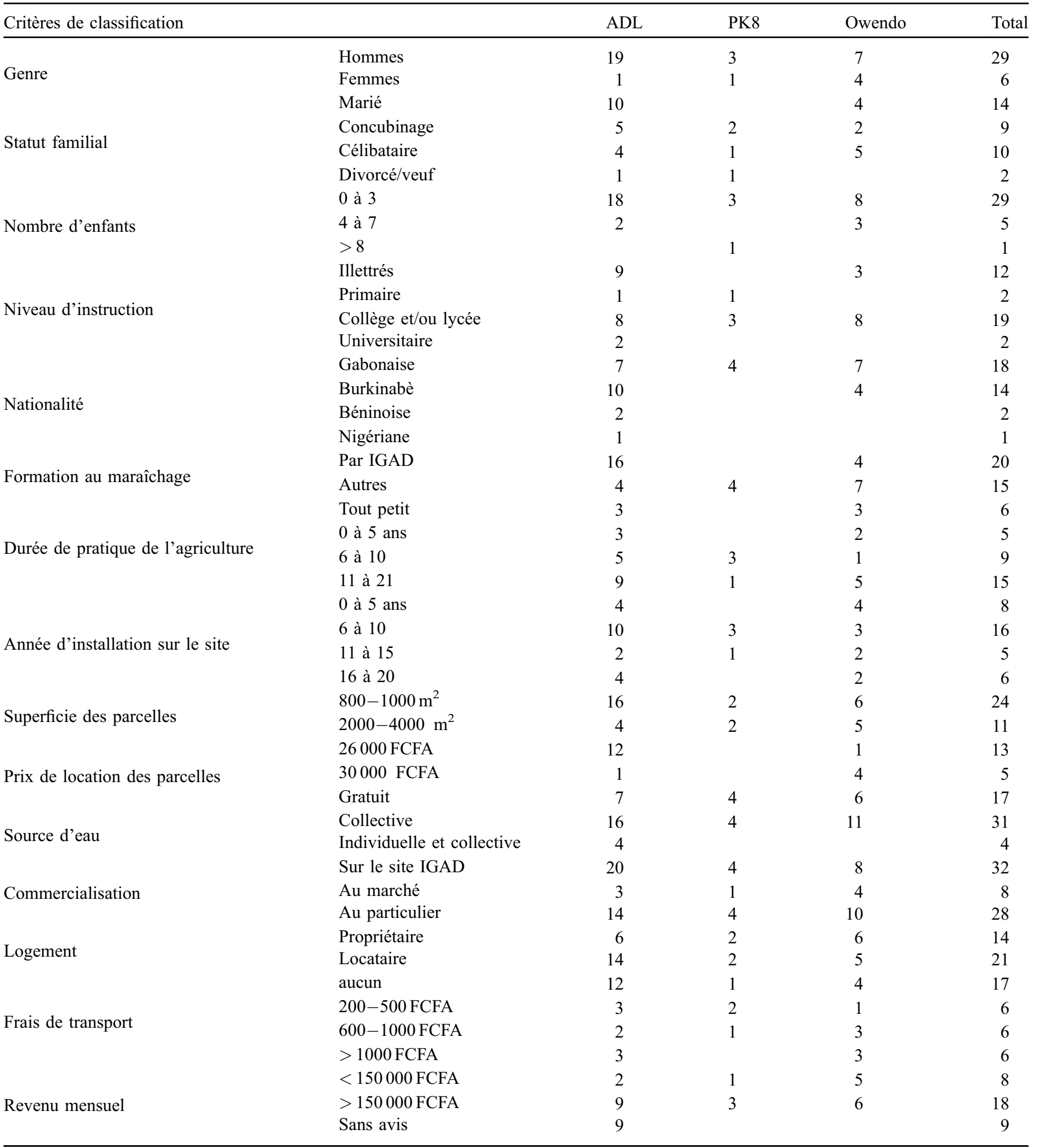


Tableau 2. Proportion de producteurs (\%) cultivant des espèces maraîchères, par saison.

Table 2. Proportion of farmers growing vegetable crops, by season.

\begin{tabular}{|c|c|c|c|c|}
\hline Cultures & Noms Scientifiques & Familles & $\begin{array}{l}\text { Saison } \\
\text { pluvieuse }\end{array}$ & $\begin{array}{l}\text { Saison } \\
\text { sèche }\end{array}$ \\
\hline Amarante & Amaranthus hybridus L. & Amarantacées & 74 & 49 \\
\hline Aubergine violette & Solanum melongena L. & Solanacées & 14 & 11 \\
\hline Baselle & Basella alba L, Basella rubra L. & Basellacées & 20 & 26 \\
\hline Basilic & Ocimum basilicum $\mathrm{L}$. & Lamiacées & 20 & 14 \\
\hline Chou pommé & Brassica oleracea var. capitata L. & Brassicacées & 0 & 6 \\
\hline Concombre & Cucumis sativus $\mathrm{L}$. & Cucurbitacées & 0 & 3 \\
\hline Corette & Corchorus olitorius L. & Liliacées & 11 & 11 \\
\hline Coriandre & Coriandrum sativum $\mathrm{L}$. & Apiacées & 6 & 3 \\
\hline Gombo & Abelmoschus esculentus (L.) Moench & Malvacées & 14 & 6 \\
\hline Oseille de guinée & Hibiscus sabdariffa L. & Malvacées & 37 & 20 \\
\hline Persil & Petroselinum crispum Mill. & Apiacées & 20 & 17 \\
\hline Piment & Capsicum frutescens $\mathrm{L}$. & Solanacées & 6 & 6 \\
\hline Poireau & Allium porrum L. & Liliacées & 0 & 3 \\
\hline Radis & Raphanus sativus L. & Brassicacées & 3 & 3 \\
\hline Tomate & Solanum lycopersicum L. & Solanacées & 9 & 11 \\
\hline
\end{tabular}

ne bénéficient pas d'un bon entretien, l'association, sous le contrôle de l'IGAD, peut attribuer un bail à des ressortissants étrangers ou à d'autres nationaux.

Tous les sites sont dotés de motopompes qui permettent d'avoir accès à l'eau. Cependant, en saison sèche, la quantité d'eau n'est plus suffisante pour tous les exploitants. Certains ne cultivent donc pas pendant cette saison-là. D'autres $(11 \%)$ s'associent pour avoir un puits commun qui leur permet de mener à bien leur culture, comme cela a été constaté sur le site d'ADL.

Il faut également noter que, depuis notre enquête, le site d'Owendo a été fermé pour des raisons politiques en relation avec l'acquisition du foncier. Tous les maraîchers ont dû arrêter leurs activités en attendant de trouver d'autres parcelles. Il serait dès lors important qu'au sein du ministère de l'Aménagement du territoire, la mise en place de terrains dédiés à l'agriculture périurbaine soit prévue pour permettre le développement économique local.

Les superficies des parcelles vont de 0,08 ha à 0,40 ha. Ces superficies sont du même ordre de grandeur que celles des parcelles maraîchères au Tchad et en République Centrafricaine $(0,1$ à $0,25 \mathrm{ha})$; celles du Cameroun peuvent être cinq fois plus grandes (0,5 à 2,0 ha; Sougnabé et al., 2010).

\subsubsection{Cultures}

La culture se fait sur des planches de 1,2 $\mathrm{m}$ de large sur $5 \mathrm{~m}$ de long en moyenne. Les exploitants disposent d'une petite zone réservée aux pépinières. Ces dernières sont en général couvertes de film plastique. Vingt-deux cultures ont été recensées, appartenant à dix familles botaniques (Tab. 2). Pour toutes les espèces cultivées, les légumes feuilles sont les plus importants $(62 \%) ; 24 \%$ des plantes sont cultivées pour leurs fruits et $14 \%$ pour leurs bulbes. Cette prédominance des légumes feuilles est également rencontrée au Togo, avec $53 \%$ des cultures (Kanda et al., 2009). Les espèces les plus exploitées, tout au long de l'année, sont l'amarante (Amaranthus hybridus L., 97\%), l'oseille de Guinée (Hibiscus sabdariffa L., $60 \%$ ), la laitue (Lactuca sativa L., $51 \%$ ), la baselle (Basella alba L., Basella rubra L., 40\%), la morelle noire (Solanum nigrum L., 40\%), le basilic (Ocimum basilicum L., 29\%), l'aubergine violette (Solanum melongena L., 26\%), le persil (Petroselinum crispum Mill., $23 \%$ ), la tomate (Solanum lycopersicum L., 20\%) et le gombo (Abelmoschus esculentus L., 20\%).

Hormis les malvacées (oseille de Guinée et gombo), les autres cultures passent par une pépinière d'au moins deux semaines, avant d'être repiquées au champ.

Les exploitants ont rarement recours à de la main-d'œuvre extérieure. Cependant, lorsqu'ils sont débordés, ils peuvent se faire aider de façon ponctuelle par des personnes à charge de leur ménage ou par des jeunes voisins, moyennant une compensation monétaire. Ce travail peut concerner aussi bien la mise en place des planches, le repiquage, le binage que le sarclage. Le commerce est strictement réservé à l'exploitant ou à un membre de sa famille.

\subsection{3 Équipement agricole}

Le matériel agricole ainsi que les intrants sont stockés dans les locaux aménagés par l'IGAD sur les sites, mais certains maraîchers construisent aussi des cabanes de rangement. La diversité et les quantités de matériel que possèdent les 


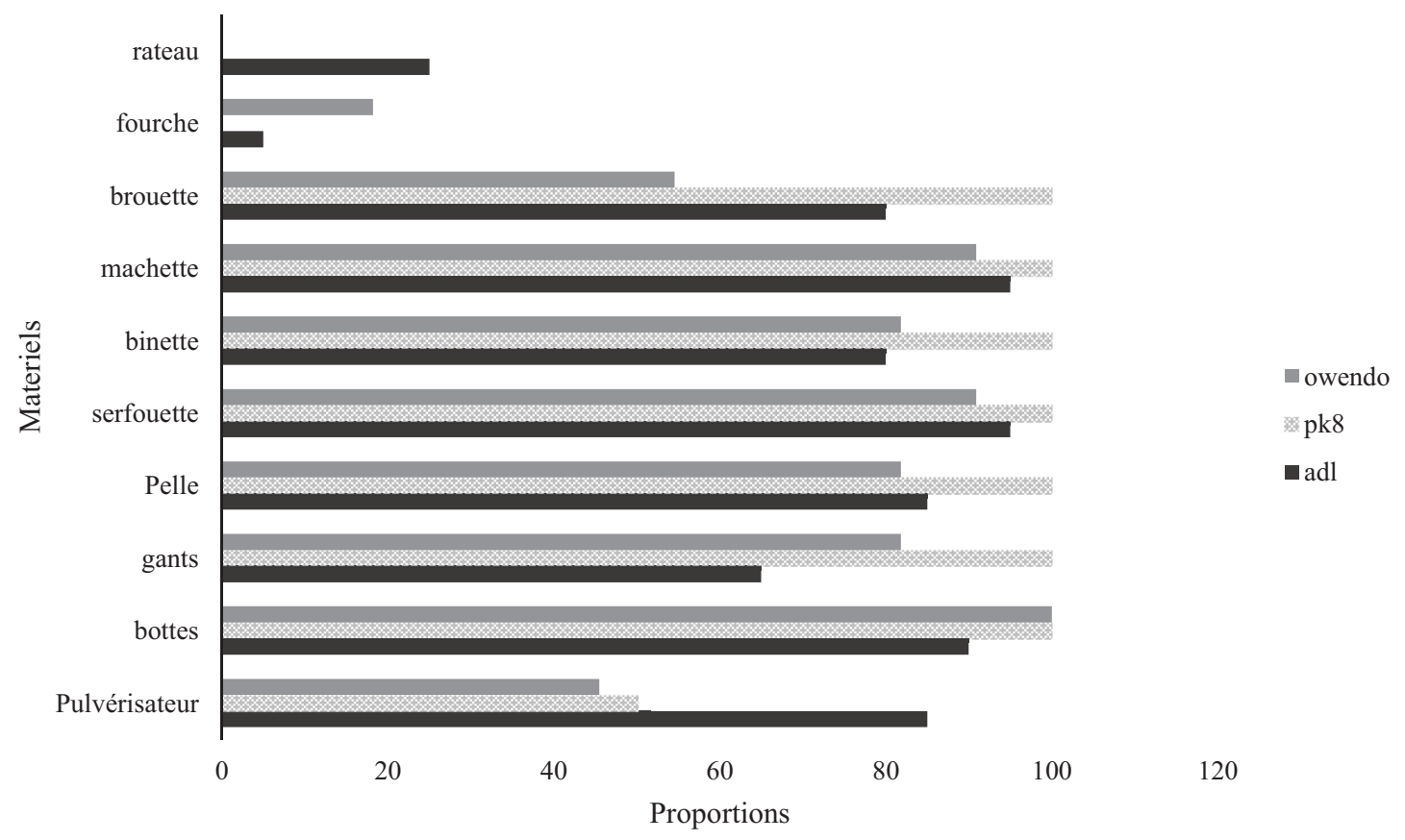

Fig. 2. Proportion d'exploitants détenant du matériel agricole, par site.

Fig. 2. Proportion of farmers owning agricultural equipment, by site.

Tableau 3. Proportion de producteurs (\%) constatant la présence de ravageurs par culture.

Table 3. Proportion of farmers (\%) confirming the presence of pests by crop.

\begin{tabular}{|c|c|c|c|c|c|c|}
\hline Cultures & Aphididae & Lépidoptères & Chrysomelidae & Autres insectes & Acariens & Maladies \\
\hline Aubergine locale (Solanum aethiopicum L.) & 6 & & & & & \\
\hline Baselle (Basella alba L., Basella rubra L.) & 6 & & & & & 11 \\
\hline Chou de chine (Brassica rapa var pekinensis (Lour.) Hanelt) & & 11 & & & & 3 \\
\hline Chou pommé (Brassica oleracea var. capitata L.) & & 6 & & & & 3 \\
\hline Coriandre (Coriandrum sativum L.) & 3 & 3 & & & & 6 \\
\hline Gombo (Abelmoschus esculentus (L.) Moench) & 9 & & 6 & & & \\
\hline Laitue (Lactuca sativa L.) & 11 & 3 & & 3 & & 9 \\
\hline Morelle noire (Solanum nigrum L.) & 17 & & & & 3 & 3 \\
\hline Oseille de guinée (Hibiscus sabdariffa L.) & 31 & & 37 & & & 3 \\
\hline
\end{tabular}

maraîchers sont présentées sur la Figure 2. Plus de la moitié des exploitants interrogés possèdent le matériel de base pour conduire efficacement les cultures, quel que soit le site. De plus, il existe une entraide qui permet le prêt de certains outils, tels que les pulvérisateurs à dos (qui doivent être différents pour des traitements herbicides d'une part et insecticides et/ou fongicides d'autre part). Certains exploitants (40\% à ADL et $9 \%$ à Owendo) ont chacun au moins deux arrosoirs car l'eau est un élément très important dans l'entretien des cultures. De façon générale, les maraîchers n'ont pas de vêtements adéquats pour les traitements chimiques. Ils utilisent cependant des bottes et des gants.

\subsection{Principaux ravageurs et utilisation des produits phytopharmaceutiques}

\subsubsection{Connaissance des ravageurs}

Les connaissances des maraîchers sur les insectes et autres agents nuisibles aux cultures sont plutôt restreintes. Leurs informations sur 15 cultures sont synthétisées au Tableau 3. Les insectes les plus connus sont les pucerons (Aphididae) sur près de 12 cultures, les coléoptères aux couleurs métalliques (Chrysomelidae) ainsi que des chenilles (larves de lépidoptères). Certains maraîchers (57\%) reconnaissent l'utilité des associations culturales sans toutefois les pratiquer, à cause de 


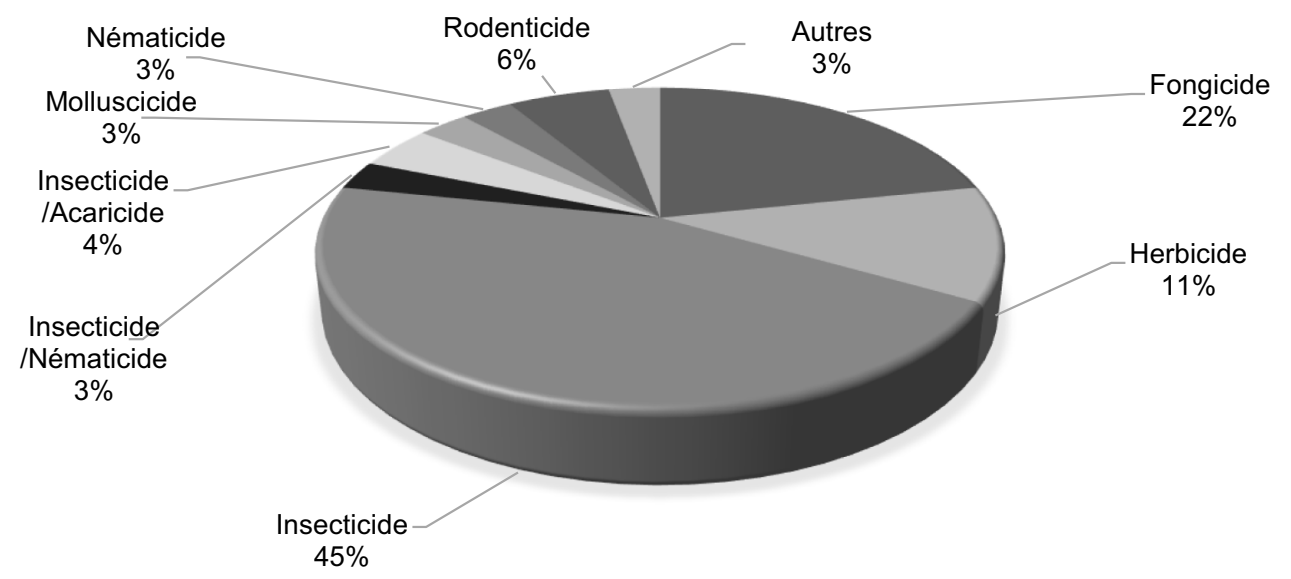

Fig. 3. Répartition des produits phytosanitaires par classes.

Fig. 3. Distribution of pesticides by classes.

la petite superficie et du type de culture (culture sur planche plutôt que par bloc).

Pour Bordat et Arvanitakis (2004), les ravageurs rencontrés au Gabon en cultures légumières sont surtout des insectes appartenant à une grande diversité de familles (Chrysomelidae, Noctuidae, Pyralidae, Pluttelidae et Thripidae). Selon Poligui (2014), les ravageurs rencontrés dans les jardins de case au Gabon appartiennent à plusieurs familles (Aphididae, Cecidomyiidae, Cicadellidae, Crambidae). Hormis ces travaux ciblés et préliminaires, aucune autre information sur les insectes nuisibles aux cultures maraîchères n'a été recensée au Gabon. Les travaux de Djiéto-Lordon et al. (2007) donnent une liste exhaustive des ravageurs inféodés aux cultures légumières au Cameroun voisin: Capsicum annuum, Lycopersicon esculentum, Solanum nigrum, Solanum aethiopicum, Solanum melongena, Abelmoschus esculentus, Corchorus olitorius, Amaranthus viridis, Phaseolus sp., Vigna sp., Glycine max, Apium graveolens, Ocimium basilicum, Ocimium sp., Lactuca sativa, Vernonia amygdalina et Cucumis melo. Pour Brévault et al. (2003), Aphis gossypii Glover, Bemisia tabaci Gennadius et Helicoverpa armigera Hübner sont des ravageurs communs des cultures maraîchères en Afrique centrale.

\subsubsection{Produits phytopharmaceutiques}

Les agents de l'IGAD ont répertorié 69 produits phytosanitaires (Fig. 3). Il y a $53 \%$ d'insecticides dont deux combinés aux acaricides et un combiné aux nématicides. Selon les entretiens réalisés avec des maraîchers, il ressort qu'il y a au total 20 pesticides disponibles sur les différents sites (Tab. 4). Il faut noter que les exploitants s'approvisionnent en pesticides selon les différents points de vente à Libreville et Owendo. Cent pour cent des exploitants du PK8 utilisent des insecticides, contre $85 \%$ à $\mathrm{ADL}$ et $82 \%$ à Owendo. Les insecticides sont essentiellement utilisés contre la teigne des crucifères sur le chou, contre les pucerons sur l'amarante, l'oseille, et le piment, et contre les chenilles sur la tomate.

La lambda-cyhalothrine est la matière active la plus employée sur tous les sites, sous les noms commerciaux de Zalang et de Karaté, sur l'amarante et la tomate pour lutter contre les chenilles et les pucerons. Les doses varient de $10 \mathrm{ml} / 101$ d'eau à $20 \mathrm{ml} / 101$ d'eau, soit de trois à six fois la dose recommandée par les guides de formation (IGAD, 2014). Selon de Bon et al. (2014), cette molécule est utilisée dans plusieurs pays africains (Tanzanie, Ghana, Bénin et Madagascar). Elle est également rencontrée au Togo (Adjrah et al., 2013).

Concernant les herbicides, $75 \%$ des maraîchers du PK8 les utilisent, alors que $55 \%$ de ceux d'Owendo le font, et que cette pratique est quasi inexistante à $\mathrm{ADL}(5 \%)$. En général, ils ont recourt aux herbicides pour l'entretien des allées autour des planches. Le paraquat et le glyphosate (herbicides totaux) sont les seules matières actives employées, à des doses variant entre $50 \mathrm{ml} / 101$ d'eau et $80 \mathrm{ml} / 10 \mathrm{l}$ d'eau.

Les fongicides sont utilisés à ADL et Owendo, par respectivement $35 \%$ et $36 \%$ des maraîchers, contre seulement $25 \%$ de ceux du PK8. Le mancozèbe et la bouillie bordelaise (sous différents noms commerciaux) sont les plus employés. La dose la plus élevée est de $30 \mathrm{ml} / 101$ d'eau, essentiellement sur le site du PK8. Les fongicides sont employés sur l'aubergine, la tomate et la baselle.

Certaines de ces matières actives sont également rencontrées en agriculture légumière en Afrique de l'Ouest: dimethoate, chlorpyrifos, malathion et glyphosate (Williamson et al., 2008).

Il n'y a pas de fréquence prédéfinie de traitement aux pesticides. Par contre, il y a au minimum deux traitements préventifs qui se font en pépinière et au champ. Les traitements curatifs interviennent dès l'apparition des symptômes d'attaques sur les plantes. Sur les parcelles de chou, il peut y avoir cinq à six applications et au moins deux sur l'amarante.

À notre connaissance, aucune étude n'a été réalisée sur les résidus de pesticides dans les sols maraîchers et les cultures du Gabon. Pour Boland et al. (2004), les risques liés à l'utilisation des pesticides et des produits chimiques en général sont plus prononcés dans les pays tropicaux que dans les pays tempérés car les effets d'empoisonnement sont plus rapides quand il fait chaud et les vêtements adaptés sont rares. Le non-respect de la réglementation par rapport à l'utilisation des pesticides (port de vêtements adéquats, fréquence, non-connaissance de délais après récolte) est noté chez la plupart des exploitants en Afrique, comme par exemple au Togo (Adjrah et al., 2013). Au Gabon, la structure chargée de l'approvisionnement et de la vente des produits phytosanitaires est la Gabonaise de chimie. $\mathrm{Au}$ niveau des différents sites, il y a des exploitants ou 
Tableau 4. Proportion d'exploitants par site utilisant les produits phytopharmaceutiques à différentes doses.

Table 4. Proportion of farmers using pesticides at different doses per site.

\begin{tabular}{|c|c|c|c|c|c|c|c|c|c|c|c|c|c|c|}
\hline Abalone $18 \mathrm{EC}$ & Abamectine $18 \mathrm{~g} / 1$ & 22 & & 6 & & & & & & & & & & \\
\hline Caldim 400 & Dimethoate $400 \mathrm{~g} / \mathrm{l}$ & 6 & & & 6 & & & & & & & & & \\
\hline Tamaron $600 \mathrm{SL}$ & Methamidophos $600 \mathrm{~g} / 1$ & 6 & & & & 9 & & & & & & & & \\
\hline Karaté 5 EC & lambda-cyhalothrine $45 \mathrm{~g} / 1$ & 39 & 11 & & 11 & 9 & & & 25 & 50 & & & & \\
\hline Malathion $50 \mathrm{EC}$ & Malathion $50 \mathrm{~g} / 1$ & & & & & & 9 & & & & & & & \\
\hline Mega $400 \mathrm{EC}$ & Dimethoate $400 \mathrm{~g} / 1$ & & & & & & 27 & & & & & & & \\
\hline Pacha 25 EC & $\begin{array}{l}\text { Lambda-cyhalothrine }(15 \mathrm{~g} / \mathrm{l}) \\
\text { Acetamipride }(10 \mathrm{~g} / \mathrm{l})\end{array}$ & 11 & & & 6 & 9 & & & & & & 25 & & \\
\hline Round up SL & Glyphosate $360 \mathrm{~g} / 1$ & & & & & & & & & & & & & 25 \\
\hline Finish $360 \mathrm{SL}$ & Glyphosate acide $360 \mathrm{~g} / 1$ & 6 & 6 & & & 9 & 18 & 18 & & & & & 25 & 25 \\
\hline \multicolumn{15}{|l|}{ Fongicide } \\
\hline Bobor WP & $\begin{array}{l}\text { Bouillie bordelaise } \\
\text { ( } 20 \% \text { de cuivre métal) }\end{array}$ & 6 & & & & & & & & & & & & \\
\hline Bouillie Bordelaise & sulfate de cuivre et chaux & & & 6 & & & & & & 25 & & 25 & & \\
\hline Cryptonol & $\begin{array}{l}\text { Sulfate double d'oxyquinoleine } \\
\text { et de potassium }\end{array}$ & & 6 & & & 9 & & & & & & & & \\
\hline Coga $80 \mathrm{WP}$ & Mancozèbe $800 \mathrm{~g} / \mathrm{kg}$ & & & & & 9 & & & & & & & & \\
\hline Manco $80 \mathrm{WP}$ & Mancozèbe $800 \mathrm{~g} / \mathrm{kg}$ & 11 & & 6 & & 18 & & & & & & 25 & & \\
\hline Pelt 44 GL & Méthylthiophanate $450 \mathrm{~g} / \mathrm{l}$ & 28 & & 6 & & & & & & & & & & \\
\hline
\end{tabular}

groupements d'exploitants qui assurent le commerce de détail des différents intrants. Toutefois, certains maraîchers utilisent des produits qui ne se sont pas forcément adaptés à l'espèce cultivée et aux conditions de cultures. À cela s'ajoute parfois le non-respect des doses et des délais avant récolte.

\subsubsection{Période et mode de traitement}

De façon générale, sur tous les sites, les traitements phytosanitaires sont pratiqués aussi bien en pépinière $(91 \%)$ qu'en culture $(60 \%)$, quel que soit le moment de la journée (Fig. 4). La grande majorité des maraîchers ne respectent pas toujours les délais avant récolte, bien qu'ils reconnaissent l'utilité de le faire. En effet, selon les guides d'information (IGAD, 2014), il est préconisé des délais avant récolte de trois ou sept jours selon les produits utilisés et la culture. Selon nos observations, certains exploitants font des traitements chimiques jusqu'à la veille des récoltes, afin de ne pas avoir d'insectes (pucerons ou chenilles) sur les produits commercialisés, nuisant ainsi à leur santé et à celle des consommateurs. Même si certains parasites semblent résistants aux traitements chimiques en cours de culture, $60 \%$ des maraîchers traitent quand même. Pour Edou Edou (2009), les maraîchers ont une méconnaissance de la période de traitement, des différents mélanges et des doses à appliquer : il arrive qu'ils fassent des mélanges de deux insecticides ou de deux fongicides dans un même pulvérisateur, que ce soit en mode préventif et/ou curatif. Parmi les producteurs interrogés, seulement $11 \%$ utilisent des combinaisons, des gants, des lunettes et des masques lors des pulvérisations. Pour Ahouangninou et al. (2011), la non-utilisation d'équipements de protection adaptés est un facteur de risque important pour les applicateurs. Les tenues et autres équipements réservés aux traitements sont rangés sans être nettoyés et repris tels quels pour les prochaines applications. Par contre, les pulvérisateurs sont nettoyés au champ.

\subsection{Méthodes alternatives à la lutte chimique}

Les maraîchers des sites de Libreville et des communes avoisinantes utilisent systématiquement les produits chimiques pour lutter contre les bio-agresseurs des plantes (Edou Edou, 2009). En l'état actuel des connaissances, il est bien difficile d'évaluer l'incidence économique des bio-agresseurs sur les cultures et par conséquent l'intérêt des traitements préventifs reste à déterminer. Le non-respect des doses et des délais avant récolte constitue un grand facteur de risque pour les consommateurs. La sécurité des exploitants pendant ou après les traitements phytosanitaires n'est pas non plus assurée, du 


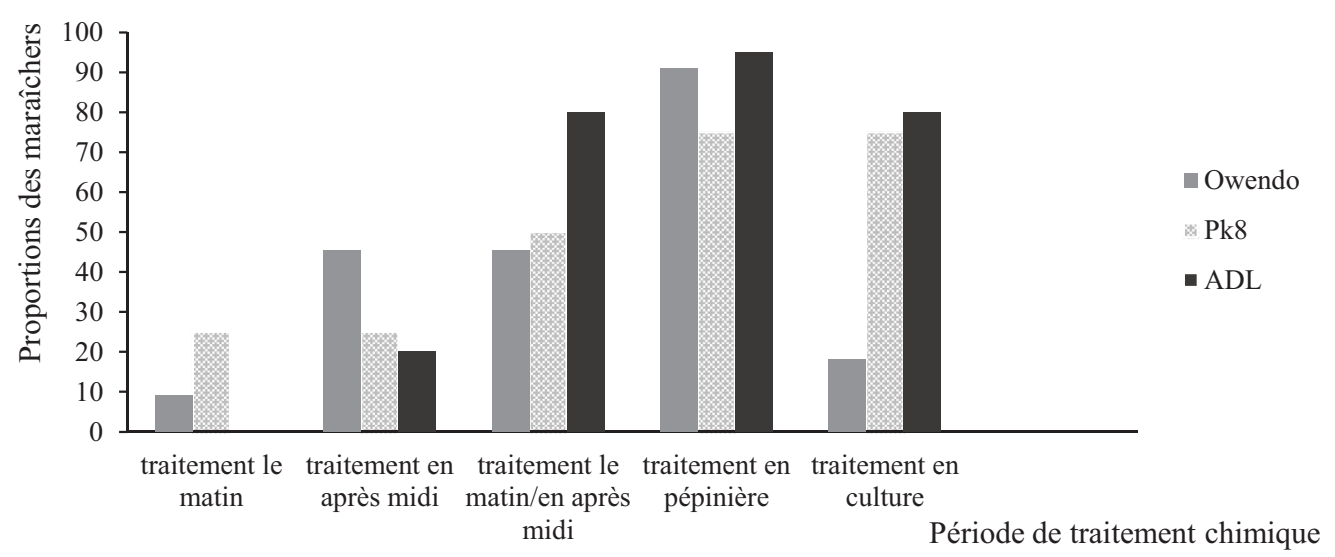

Fig. 4. Proportion de producteurs par site et par période de traitement chimique.

Fig. 4. Proportion of farmers by site and by period of chemical treatment.

fait de leur non-protection. Mettre au point des méthodes alternatives de protection des cultures permettrait de limiter le recours systématique aux pesticides et donc de préserver l'état de santé des maraîchers et des consommateurs.

Des méthodes physiques, comme les toiles moustiquaires sur le choux et la tomate, sont déjà utilisées au Bénin (Martin et al., 2015; Vidogbéna et al., 2015). La lutte biologique, avec l'emploi de certains champignons entomopathogènes comme Beauveria bassiana (Bals.-Criv.), Metarhizium anisopliae (Metchnikoff) et des ennemis naturels (de Bon et al., 2014) pourrait aussi limiter la présence des ravageurs. De plus, certaines plantes à fleurs pourraient aussi être implantées autour des champs pour favoriser la présence des auxiliaires. Des études récentes envisagent aussi l'utilisation de répulsifs (Deletre et al., 2016), et d'huiles essentielles (Zoubiri et Baaliouamer, 2014). Cependant, ces diverses méthodes restent à tester en conditions réelles au Gabon.

\section{Conclusion}

Bien que la Gabonaise de chimie organise des séminaires sur l'utilisation des produits chimiques pour les producteurs, ceux-ci respectent peu souvent les bonnes pratiques. L'organisme responsable des contrôles phytosanitaires du ministère de l'Agriculture devrait donc effectuer des contrôles plus fréquents pour limiter certains agissements abusifs. L'IGAD pourrait aussi avoir un volet phytosanitaire pour assurer un meilleur suivi et une meilleure formation des exploitants dont il a la charge. Cela permettrait de rassurer les consommateurs sur la qualité sanitaire des produits. Enfin, jusqu'à maintenant, rares sont les études qui ont été réalisées sur les inventaires des ravageurs des différentes cultures, les résidus de pesticides dans les plantes cultivées, dans le sol et les eaux de ruissellement; un effort de recherche serait donc à faire dans ces domaines.

De leur côté, les producteurs interrogés ont des attentes : la possibilité d'obtenir des micro-crédits et/ou du matériel adéquat. Pour limiter l'utilisation des produits chimiques, tous les producteurs sont ouverts à l'éventualité d'employer des produits d'origine naturelle pour mieux contrôler les bioagresseurs, pour autant que les prix restent acceptables. Ils sont également favorables aux formations techniques qui leur permettraient de mieux gérer leur travail.
Les résultats de cette enquête mériteraient d'être confrontés à des enquêtes complémentaires réalisées dans d'autres zones périurbaines, chez des maraîchers encadrés ou non par l'IGAD. Cela permettrait d'avoir une vue plus générale du maraîchage dans tout le pays.

Une surveillance précise des bio-agresseurs et des essais de traitements des principales cultures maraîchères (amarante, laitue, morelle noire, oseille de guinée et chou), dans une approche de gestion intégrée, devraient être réalisés dans les zones périurbaines. Les résultats obtenus pourraient alors être vulgarisés pour permettre aux maraîchers de produire des légumes de façon plus durable, et en toute sécurité pour les consommateurs et l'environnement.

\section{Matériel supplémentaire}

Annexe 1. Détails des caractéristiques socio-économiques des maraîchers de Libreville et d'Owendo.

Le matériel supplémentaire est disponible sur http://www. cahiersagricultures.fr/10.1051/cagri/2017026/olm.

Remerciements. Ce travail a été effectué grâce à une convention de collaboration entre Gembloux Agro-Bio Tech (université de Liège en Belgique), l'Institut de recherches agronomiques et forestières (IRAF) et l'Institut gabonais d'appui au développement (IGAD) au Gabon. Les auteurs remercient tout le personnel et les maraîchers de l'IGAD. Nos remerciements vont également à Andeime Eyene Myrianne Flore (IRAF), Abaga Obiang Ernest (IRAF), Mbeng Hendrix (IRAF) et Poligui René Noël (INSAB) pour leur collaboration.

\section{Références}

Adjrah Y, Dovlo A, Karou SD, Eklu-Gadegbeku K, Agbonon A, de Souza C, et al., 2013. Survey of pesticide application on vegetables in the Littoral area of Togo. Annals of agricultural and environmental medicine 20(4): 715-720.

Ahouangninou C, Fayomi BE, Martin T. 2011. Évaluation des risques sanitaires et environnementaux des pratiques phytosanitaires des producteurs maraîchers dans la commune rurale de Tori-Bossito (Sud-Bénin). Cahiers Agricultures 20(3): 216-222. 
Boland J, Koomen I, van Lidth de Jeude J, Oudejans J. 2004. Les pesticides: composition, utilisation et risques. Wageningen: Agrodok, 124 p.

Bordat D, Arvanitakis L. 2004. Arthropodes des cultures légumières d'Afrique de l'Ouest, centrale, Mayotte et Réunion. Montpellier : CIRAD-FLHOR ed., 291 p.

Brévault T, Beyo J, Nibouche S, Vaissayre M. 2003. La résistance des insectes aux insecticides Problématique et enjeux en Afrique centrale. In: Savanes Africaines: des espaces en mutation, des acteurs face à de nouveaux défis. Actes du colloque, mai 2002, Garoua, Cameroun. Prasac, N'Djamena, Tchad-Cirad, Montpellier, France. http://hal.cirad.fr/PRASAC/hal-00142447.

de Bon H, Huat J, Parrot L, Sinzogan A, Martin T, Malézieux E, et al. 2014. Pesticide risks from fruit and vegetable pest management by small farmers in sub-Saharan Africa. A review. Agron Sustain Dev 34(4): 723-736.

Deletre E, Schatz B, Bourguet D, Chandre F, Williams L, Ratnadass A, et al. 2016. Prospects for repellent in pest control: current developments and future challenges. Chemoecology 26(4): $127-$ 142. Doi: 10.1007/s00049-016- 0214-0.

Direction générale des statistiques, 2010. Annuaire statistique du Gabon 2004-2008. Disponible sur http:/www.stat-gabon.org/ documents/PDF/Donnees\%20stat/Compteannuaire/Annu0408.pdf.

Djiéto-Lordon C, Alene DC, Reboul JL. 2007. Contribution à la connaissance des insectes associés aux cultures maraîchères dans les environs de Yaoundé - Cameroun. Cameroon J Biol Biochem Sci 15: 1-13.

Edou Edou G. 2009. Le système de suivi technico-économique en 2008: analyse des indicateurs de l'activité maraîchère en zone urbaine et périurbaine de Libreville. Gabon : IGAD Libreville, $81 \mathrm{p}$.

FAO. 2015. FAOSTAT comparer données. Disponible sur http:// faostat3.fao.org/home/F.

IGAD. 2014. Institut gabonais d'appui au développement. Disponible sur http://igad-gabon.com/.

James B, Atcha-Ahowé C, Godonou I, Baimey H, Goergen G, Sikirou $\mathrm{R}$, et al. 2010. Gestion intégrée des nuisibles en production maraîchère: guide pour les agents de vulgarisation en Afrique de l'Ouest. Ibadan, Nigéria: Institut international d'agriculture tropicale (IITA) ed., $120 \mathrm{p}$.

Kanda M, Wala K, Batawila K, Djaneye-Boundjou G, Ahanchede A, Akpagana K. 2009. Le maraîchage périurbain à Lomé : pratiques culturales, risques sanitaires et dynamiques spatiales. Cahiers Agricultures 18(4): 356-363. Doi: 10.1684/agr.2009.0319.

Lefort L. 2015. Statistiques mondiales. Disponible sur http://www. statistiques-mondiales.com/gabon.htm.

Martin T, Simon S, Parrot L, Komlan FA, Vidogbena F, Adegbidi A, et al. 2015. Eco-friendly nets to improve vegetable production and quality in sub-Saharan Africa. Acta Horticulturae (1105): 221-228. Doi: 10.17660/ActaHortic.2015.1105.31.

Nchoutnji I, Nzossié EJF, Bassala JO, Temple L, Kameni A. 2009. Systèmes maraîchers en milieux urbain et périurbain des zones Soudano-sahélienne et Soudano-guinéenne du Cameroun: cas de Garoua et Ngaoundéré. Tropicultura 27(2): 98-104.

Nguema R-M, 2005. Développement de la ville, découpage et appropriation des territoires urbains au Gabon : le cas de Libreville. Belgeo 4: 481-498.

Ondo JA. 2011. Vulnérabilité des sols maraîchers du Gabon (région de Libreville) : acidification et mobilité des éléments métalliques. Thèse de doctorat, université de provence France.

Organisation mondiale du commerce. 2013a. Examen des politiques commerciales : rapport des pays de la Communauté économique et monétaire de l'Afrique centrale (CEMAC). Disponible sur https:// www.wto.org/french/tratop f/tpr f/g285 f.pdf.

Organisation mondiale du commerce. 2013b. WT/TPR/S/285 Annexe 3 Gabon. In : Examen des politiques commerciales: Cameroun, Congo, Gabon, République Centrafricaine et Tchad. 262-316. Disponible sur https://www.wto.org/french/tratop_f/tpr_f/ s285-02 f.pdf.

Poligui RN $\bar{N}$. 2014. Étude de l'entomofaune au sein d'associations culturales comprenant le Safoutier (Dacryodes edulis) dans le Haut-Ogooué (Gabon). Thèse de doctorat. Gembloux université de Liège Belgique.

Sougnabé SP, Yandia A, Acheleke J, Brevault T, Vaissayre M, Ngartoubam LT. 2010. Pratiques phytosanitaires paysannes dans les savanes d'Afrique centrale, actes du colloque "Savanes africaines en developpement: innover pour durer ", 20-23 Apr 2009, Garoua, Cameroon. Cirad, http://hal.cirad.fr/PRASAC2009/ cirad-00471372.

Temple L, Moustier P. 2004. Les fonctions et contraintes de l'agriculture périurbaine de quelques villes africaines (Yaoundé, Cotonou, Dakar). Cahiers Agricultures 13(1): 15-22.

Vidogbéna F, Adégbidi A, Tossou R, Assogba-Komlan F, Martin T, Ngouajio M, et al. 2015. Consumers' willingness to pay for cabbage with minimized pesticide residues in Southern Benin. Environments 2(4): 449-470. Doi: 10.3390/environments2040449.

Williamson S, Ball A, Pretty J. 2008. Trends in pesticide use and drivers for safer pest management in four African countries. Crop Protection 27(10): 1327-1334. Doi: 10.1016/j.cropro.2008.04.006.

WRI (World Resources Institute), 2013. The Interactive forest atlas for Gabon. Available from http://www.wri.org/our-work/project/ congo-basin-forests/gabon\#project-tabs (16/06/2013).

Zoubiri S, Baaliouamer A. 2014. Potentiality of plants as source of insecticide principles. Journal of Saudi Chemical Society 18(6): 925-938. Doi: 10.1016/j.jscs.2011.11.015.

Citation de l'article : Bayendi Loudit SM, Ndoutoume Ndong A, Francis F. 2017. Le maraîchage périurbain à Libreville et Owendo (Gabon) : pratiques culturales et durabilité. Cah. Agric. 26: 45002. 